Rapid Reviews COVID-19

\title{
Reviews of "Preprinting a pandemic: the role of preprints in the COVID-19 pandemic"
}

Noah Haber ${ }^{1}$, Emily Smith ${ }^{2}$, Siran $\mathrm{He}^{2}$

${ }^{1}$ Meta Research Innovation Center at Stanford University,

${ }^{2}$ Department of Global Health, George Washington University

Published on: Aug 11, 2020

DOI: $10.1162 / 2 \mathrm{e} 3983 f 5 . d 9 f 18647$

License: Creative Commons Attribution 4.0 International License (CC-BY 4.0). 
To read the original manuscript, click the link above.

Summary of Reviews: This study proves that COVID-19 has led to the unprecedented role of preprints and preprint servers in the dissemination of COVID-19 science.

Findings are robust and informative, though there are some errors and misinterpretations.

\section{Reviewer 1 (Noah Haber)}

Reviewer 2 (Emily Smith, Siran He) |

\section{RR:C19 Strength of Evidence Scale Key}

प्रमप = Misleading

प्र

प्रा पि = Potentially Informative

प्राप्र = Reliable

प्राप्र = Strong

To read the reviews, click the links below. 